\title{
Signaling through hepatocellular A2B adenosine receptors dampens ischemia and reperfusion injury of the liver
}

\author{
Michael A. Zimmerman ${ }^{a, 1}$, Almut Grenz ${ }^{\mathrm{b}, 1}$, Eunyoung Tak ${ }^{\mathrm{b}, 1}$, Maria Kaplan $^{\mathrm{a}}$, Douglas Ridyard ${ }^{\mathrm{b}}$, Kelley S. Brodsky ${ }^{\mathrm{b}}$, \\ Mercedes Susan Mandell ${ }^{\mathrm{b}}$, Igal Kam ${ }^{\mathrm{a}}$, and Holger K. Eltzschig ${ }^{\mathrm{b}, 2}$ \\ aDivision of Transplant Surgery, Department of Surgery, and b ${ }^{b}$ Mucosal Inflammation Program, Department of Anesthesiology, University of Colorado, \\ Denver, Aurora, CO 80045
}

Edited by Fadi Lakkis, University of Pittsburgh, Pittsburgh, PA, and accepted by the Editorial Board May 17, 2013 (received for review December 13, 2012)

Ischemia and reperfusion significantly contributes to the morbidity and mortality of liver surgery and transplantation. Based on studies showing a critical role for adenosine signaling in mediating tissue adaptation during hypoxia, we hypothesized that signaling events through adenosine receptors (ADORA1, ADORA2A, ADORA2B, or ADORA3) attenuates hepatic ischemia and reperfusion injury. Initial screening studies of human liver biopsies obtained during hepatic transplantation demonstrated a selective and robust induction of ADORA2B transcript and protein following ischemia and reperfusion. Subsequent exposure of gene-targeted mice for each individual adenosine receptor to liver ischemia and reperfusion revealed a selective role for the Adora2b in liver protection. Moreover, treatment of wild-type mice with an Adora2b-selective antagonist resulted in enhanced liver injury, whereas Adora2b-agonist treatment was associated with attenuated hepatic injury in wild-type, but not in Adora $2 b^{-/-}$mice. Subsequent studies in mice with $A d o r a 2 b$ deletion in different tissues-including vascular endothelia, myeloid cells, and hepatocytes-revealed a surprising role for hepatocellular-specific Adora2b signaling in attenuating nuclear factor NF-KB activation and thereby mediating liver protection from ischemia and reperfusion injury. These studies provide a unique role for hepatocellularspecific Adora2b signaling in liver protection during ischemia and reperfusion injury.

$\mathbf{H}$ epatic ischemia and reperfusion injury is an important source of morbidity and mortality during major liver surgery and hepatic transplantation $(1,2)$. Extracellular adenosine is a signaling molecule that has been implicated in attenuating inflammation during conditions of hypoxia or ischemia (3-5). Indeed, conditions of limited oxygen availability—such as hepatic ischemia-are associated with increased production of adenosine from precursor nucleotides, such as ATP, ADP, or AMP $(6,7)$. Hypoxia and ischemia are associated with the transcriptional induction of the ectoapyrase CD39 (extracellular conversion of ATP/ADP to AMP) (8), and $c d 39^{-/-}$mice are more prone to hepatic ischemia and reperfusion, whereas CD39 is also critical for liver regeneration (9). Moreover, hypoxia also drives the transcriptional induction of the ecto-5' nucleotidase CD73 (conversion of AMP to adenosine), and gene-targeted mice for $c d 73$ experience a more severe phenotype in models of hepatic ischemia and reperfusion (10), thus suggesting a protective role of extracellular adenosine generation during hepatic ischemia and reperfusion injury. Based on these findings, we hypothesized that extracellular receptors for adenosine could represent therapeutic targets for attenuating hepatic ischemia and reperfusion injury. At present, the functional role of individual adenosine receptors (ARs), their tissue-specific roles, and the relevance of the adenosine-signaling pathway during human liver ischemia and reperfusion injury remain unknown. To make progress on these issues, we combined studies of liver biopsies in patients undergoing liver transplantation with pharmacologic and genetic studies in mice. Indeed, our findings point toward a protective role of adenosine receptor $2 b$ (Adora2b) signaling on hepatocytes in liver protection.

\section{Methods}

Human Liver Tissue. Liver samples were obtained from patients undergoing orthotopic liver transplantation (Fig. 1A, SI Methods, and Table S1).

Murine Model of Partial Liver Ischemia. A murine model of partial liver ischemia was employed using a hanging-weight system as previously described (SI Methods) (11).

Transcriptional Analysis and Immunoblotting. Transcript levels and protein content were measured as previously described (SI Methods and Tables S2 and S3) (12-14).

ELISA and Assays. IL-6 and TNF $\alpha$ (R\&D Systems), neutrophil sequestration, transcriptional activity of NF-KB and CAMP was quantified as previously described (see SI Methods) $(14,15)$.

Liver Histology. Liver tissue was stained as previously described (SI Methods) (10).

Isolation of Hepatocytes, Endothelial Cells, and Macrophages. Specific cell isolation was performed as described previously (SI Methods) (16-18).

Adora2b Genetic Deletion in Cell Cultures. Human hepatocytes (HepG2) were obtained from ATCC and cells were cultured and transfected as previously described (SI Methods) (10).

Statistical Analysis. Liver injury score data are given as median and range. All other data are presented as mean \pm SD from three to eight animals per condition. We performed statistical analysis using the Student's $t$ test. A value of $P<0.05$ was considered statistically significant. For Western blot analysis three repeats were performed. For all statistical analysis GraphPad Prism 5.0 software for Windows XP was used.

Study Approval. Collection and use of patient samples were approved by the Colorado Multiple Institutional Review Board (COMIRB) at University of Colorado, Denver (COMIRB Protocol 10-0100) and informed consent was obtained. All animal protocols were in accordance with the US guidelines by the in stitutional animal care and use committee for use of living animals and were approved by the Institutional Animal Care and Use Committee of the University of Colorado guidelines for animal care.

\section{Results}

Selective Induction of Human Adora2b Transcript and Protein Levels During Hepatic Ischemia and Reperfusion Injury. Previous studies had indicated that the enzymatic control of extracellular adenosine contributes to liver protection from ischemia and reperfusion $(9$,

Author contributions: A.G., K.S.B., and H.K.E. designed research; M.A.Z., A.G., E.T., M.K. D.R., and M.S.M. performed research; M.S.M. and I.K. contributed new reagents/analytic tools; M.A.Z., A.G., E.T., and D.R. analyzed data; and A.G. and H.K.E. wrote the paper. The authors declare no conflict of interest.

This article is a PNAS Direct Submission. F.L. is a guest editor invited by the Editorial Board. ${ }^{1}$ M.A.Z., A.G., and E.T. contributed equally to this work.

${ }^{2}$ To whom correspondence should be addressed. E-mail: Holger.Eltzschig@UCDenver.edu. This article contains supporting information online at www.pnas.org/lookup/suppl/doi:10 1073/pnas.1221733110/-/DCSupplemental. 
A

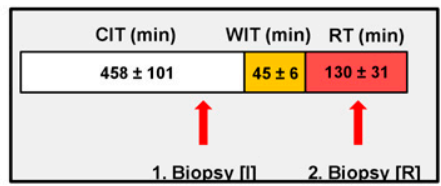

B
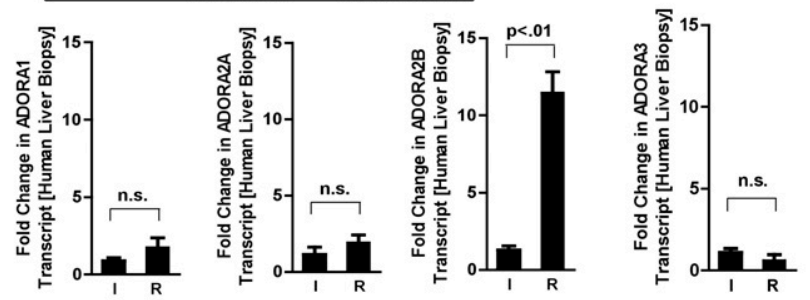

C

$$
\frac{P 1}{I R} \frac{P 2}{I R} \frac{P 3}{I R} \frac{P 4}{I R} \frac{P 5}{I R}
$$

ADORA2B

$\beta$-actin

D

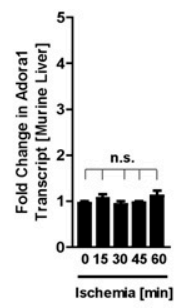

$\mathrm{E}$
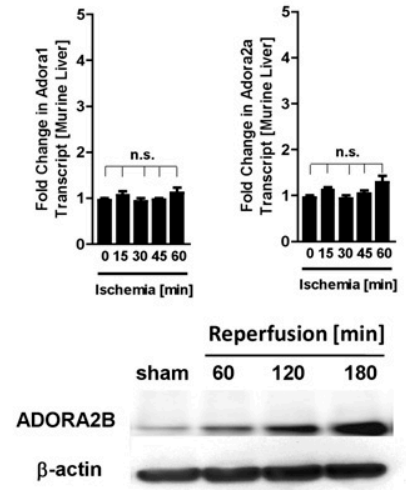
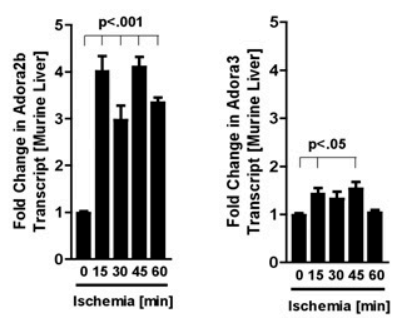

Fig. 1. Adenosine receptor expression in biopsies of human liver transplants and murine livers following hepatic ischemia and reperfusion. $(A)$ First liver biopsy was taken during ischemia (I) at the conclusion of cold ischemia time (CIT) during back table preparation of the cadaveric liver allograft. A second biopsy was taken during reperfusion (R) after warm ischemia time (WIT) and reperfusion time (RT) immediately before closure of the abdomen following drain placement. $(B)$ ADORA1, ADORA2A, ADORA2B, and ADORA3 transcript levels in biopsies of human liver transplants during cold ischemia (I) and after reperfusion (R); ( $n=3-4$ independent experiments). (C) ADORA2B protein levels (one representative blot of three is shown). (D) Adora1, Adora2a, Adora2b, and Adora3 transcript levels in left livers exposed to sham operation ( 0 ) or $15,30,45$, and $60 \mathrm{~min}$ of ischemia followed by $2 \mathrm{~h}$ reperfusion ( $n=$ $3-4$ independent experiments). (E) Adora2b protein levels ( $\beta$-actin to control for loading conditions; one representative blot of three is shown).

10). Based on these studies, we hypothesized that signaling events through individual adenosine receptors (ARs) could function to dampen hepatic ischemia and reperfusion injury. To address this hypothesis and to gain insight into the transcriptional regulation of ARs during ischemia and reperfusion injury of the liver in humans, we included five patients undergoing liver transplantation in a small clinical trial (COMIRB Protocol 10-0100). Characteristics of the recipients and donors are summarized in Table S1. For the purpose of this study, we performed two subsequent liver biopsies in the course of the liver transplantation surgery (Fig. 1A). The first biopsy (ischemia, I) was obtained following graft procurement and cooling [cold ischemia only, average cold ischemia time (CIT) $453.2 \pm 101.1$ min; Table S1 and Fig. 1A]. A subsequent biopsy (reperfusion, $\mathrm{R}$ ) of the same liver was obtained by the end of the surgery following exposure to warm ischemia and reperfusion [average warm ischemia time (WIT) $44.8 \pm 6.3 \mathrm{~min}$; average reperfusion time $129.6 \pm 31.4 \mathrm{~min}$; Table S1, Fig. $1 A$ ]. Each liver served as its own control. Surprisingly, we found a very robust and selective induction of the ADORA2B (over 10-fold induction; $P<0.001$ ), whereas transcript levels of other ARs were unchanged (Fig. 1B). Moreover, we found dramatic increases of ADORA2B protein levels following warm ischemia and reperfusion for each individual patient (Fig. $1 C$ ). Taken together, these studies uniquely indicate that hepatic ischemia and reperfusion is associated with a selective induction of the ADORA2B during human liver transplantation.

Global Deletion of Adora2b Aggravates Murine Ischemia and Reperfusion Injury of the Liver. Consistent with the above studies during human liver transplantation, we observed a selective induction of Adora2b transcript and protein levels following hepatic ischemia (Fig. $1 D$ and $E$ ). As next step, we examined previously described mice with genetic deletion for individual ARs during hepatic ischemia and reperfusion (12). Indeed, mice gene targeted for Adora1 (Fig. 2A), Adora2A (Fig. 2B), or Adora3 (Fig. 2D) had similar degrees of hepatic tissue injury as corresponding littermate controls matched in weight, age, and sex exposed to $45 \mathrm{~min}$ of partial hepatic ischemia followed by $2 \mathrm{~h}$ of reperfusion. In contrast, $A$ dora $2 b^{-/-}$mice exhibited significantly higher levels of tissue injury as examined by elevations of the transaminases aspartate transaminase (AST) and alanine transaminase (ALT) and liver histology (Fig. 2C). To correlate Adora2b expression levels with hepatic injury, we measured cAMP levels and hepatic injury as a readout for Adora2b signaling in wild-type, Adora $2 b^{+/-}$, and Adora $2 b^{-/-}$mice. Consistent with previous studies on Adora2a signaling (18), we found that hepatic cAMP levels inversely correlate with expression levels for Adora2b signaling: cAMP levels are highest in postischemic livers of wild-type mice, in-

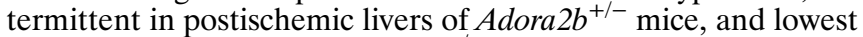
in postischemic livers of $A d o r a 2 b^{-/-}$mice (Fig. S1A). Moreover, the cAMP levels in these three groups inversely correlate with a more severe liver injury following ischemia and reperfusion injury (Fig. $\mathrm{S} 1 B$ ), indicating that there is no reserve of Adora2b signaling (e.g., no spare receptors). Along these lines, different markers of liver inflammation (IL-6, TNF $\alpha$, hepatic neutrophil accumulation assessed by myeloperoxidase (MPO) measurements; Fig. S1C) were increased in $A d o r a 2 b^{-1-}$ mice compared with controls following liver ischemia and reperfusion injury. Moreover, second organ injury as examined by increases of IL-6 or myeloperoxidase in the lungs was significantly enhanced in gene-targeted mice for the Adora2b (Fig. S1D). In addition we performed experiments with prolonged reperfusion times. In these experiments, we followed 45 min of liver ischemia with $24 \mathrm{~h}$ of reperfusion. Indeed, Adora $2 b^{-1-}$ mice exhibited significantly higher levels of tissue injury as examined by elevations of the transaminases AST and ALT (Fig. S1E) and liver histology (Fig. $\mathrm{S} 1 F$ ) after $24 \mathrm{~h}$ of reperfusion time. Taken together, these studies in gene-targeted mice for individual ARs implicate a selective role for the Adora2b in liver protection from ischemia and reperfusion injury. Furthermore these findings highlight that Adora2b receptor expressional levels correlate with Adora2bmediated tissue protection from ischemia and reperfusion injury.

Pharmacologic Inhibition of Adora2b Aggravates Hepatic Ischemia and Reperfusion Injury. After having shown that genetic deletion of the Adora2b is associated with more severe hepatic injury and liver inflammation following ischemia and reperfusion, we next pursued pharmacologic studies with a previously described Adora2b antagonist 1-propyl-8-(p-sulfophenyl) xanthine (PSB1115). For the purpose of these studies, we administered PSB1115 30 min before the onset of ischemia via retroorbital injection $(0.5 \mathrm{mg} / 25 \mathrm{~g}$ mouse i.v.). Consistent with the previous studies in gene-targeted mice for $A d o r a 2 b$, we observed elevated liver injury as assessed by elevations of transaminases (Fig. S2A), histologic tissue injury (Fig. S2B), liver inflammation (Fig. S2C), and second organ injury (Fig. S2D). Moreover, the observed alterations of liver injury following PSB1115 treatment were abolished in gene-targeted mice for Adora $2 b$ (Fig. S2E), thereby providing evidence for the specificity of PSB1115 for Adora2b. Together with the studies in Adora $2 b^{-/-}$ 


\section{$\square$ Adora1 $^{+/+} \square$ Adora1 $\%$}
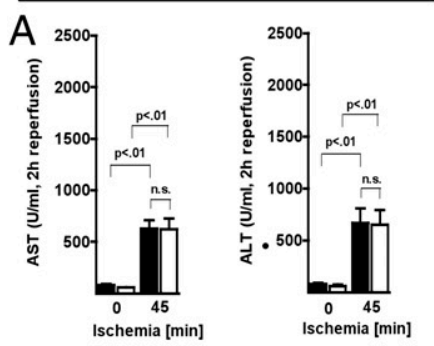

$\square$ Adora2a $^{+/+} \square$ Adora2a $^{-/-}$
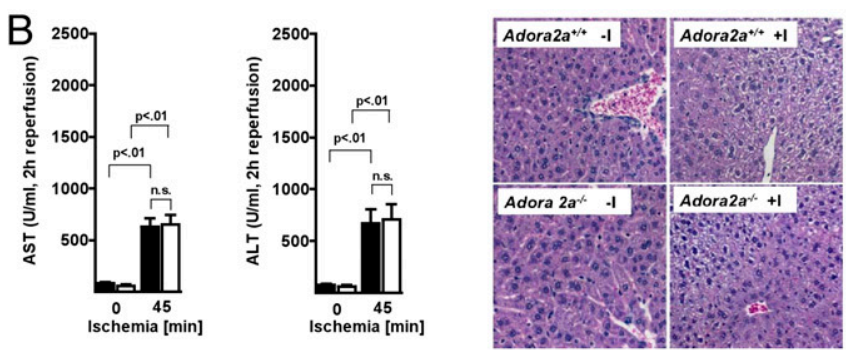

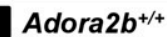

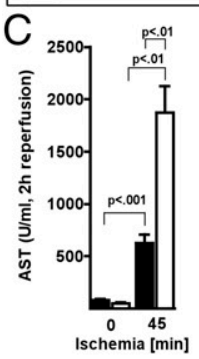

$\square$ Adora2b $^{-/}$
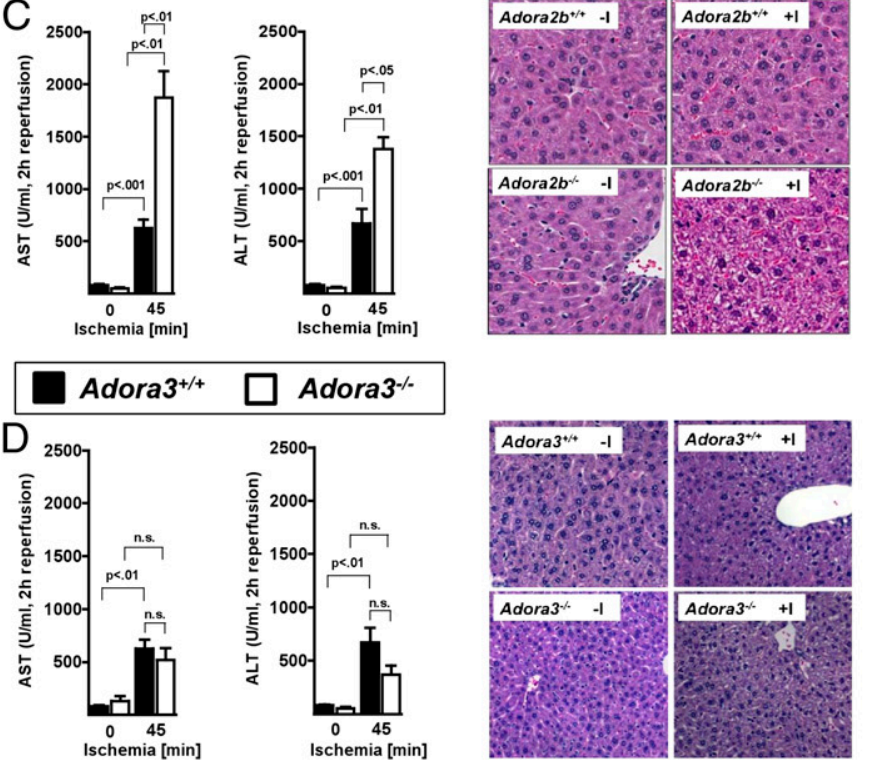

mice, these findings indicate that inactivation of Adora2b via genetic deletion or pharmacologic inhibition significantly aggravates liver injury following hepatic ischemia and reperfusion.

Treatment with Adora2b Agonist Provides Potent Protection from Hepatic Ischemia and Reperfusion Injury. After having shown that targeted deletion or specific inhibition of the Adora2b is associated with enhanced tissue injury, we next examined a potential therapeutic role for Adora2b agonist treatment during liver ischemia and reperfusion. Indeed, we recently described a specific and effective Adora2b agonist [2-[6-amino-3,5-dicyano-4[4-(cyclopropylmethoxy)phenyl]pyridin-2-ylsulfanyl]acetamide (BAY 60-6583)] (12). For the purpose of these studies, BAY $60-6583$ ( $0.25 \mathrm{mg} / 25 \mathrm{~g}$ mouse i.v.) was administered via retroorbital injection $30 \mathrm{~min}$ before the induction of liver ischemia for $45 \mathrm{~min}$ and $2 \mathrm{~h}$ reperfusion. Indeed, Adora2b agonist treatment was associated with a marked reduction in liver injury and inflammation as assessed by plasma levels of transaminases AST,
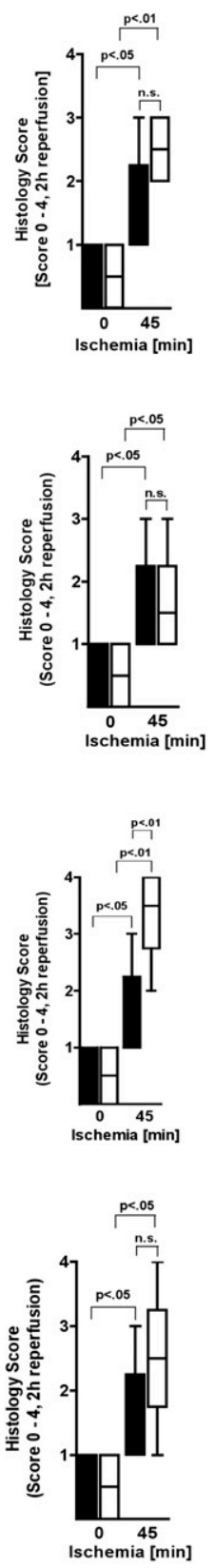

Fig. 2. Ischemic liver injury in mice gene targeted for Adora1, Adora2a, Adora2b, or Adora3 adenosine receptor. (A) Adora1 ${ }^{-1-},(B)$ Adora2a $^{-1-},(C)$

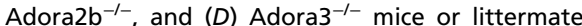
controls were exposed to $45 \mathrm{~min}$ of liver ischemia and $2 \mathrm{~h}$ of reperfusion before liver function was assessed by measurement of AST, ALT, liver histology (200-fold magnification; one of three representative slides is shown) and quantification of histologic tissue injury (Suzuki scoring index, 0-4) $(n=4-6)$

ALT (Fig. S3A), hepatic histology (Fig. S3B), liver inflammation (Fig. S3C), and second organ injury of the lungs (Fig. S3D). Additional studies in gene-targeted mice for Adora $2 b$ revealed that the protective effects of BAY 60-6583 are completely abolished in Adora $2 b^{-/-}$mice, thereby providing evidence for the specificity of BAY 60-6583 for Adora2b (Fig. S3E). To test the long-term effect of liver protection via Adora2b agonist (BAY 60-6583) treatment, we performed experiments with $24 \mathrm{~h}$ reperfusion following $45 \mathrm{~min}$ of liver ischemia. Indeed, Adora2b agonist treatment provided similar protection after $24 \mathrm{~h}$ of reperfusion as indicated by a robust reduction in plasma levels of transaminases AST, ALT, and hepatic histology (Fig. S4 $A$ and $B$ ). Together, these studies provide evidence that Adora2b agonist treatment is therapeutic during liver ischemia and reperfusion injury.

Identification of Hepatocyte-Specific Adora2b Signaling in Liver Protection from Ischemia and Reperfusion Injury. As the next step, we pursued studies to address the tissue-specific contributions of 
Adora2b signaling in hepatic ischemia and reperfusion injury. Indeed, we used a recently described transgenic mouse line with a "floxed" Adora $2 b$ gene (12). We used this mouse line to generate mice with tissue-specific deletions of Adora2b in different cellular compartments of the liver. As such we generated mice with endothelial Adora $2 b$ deletion (Adora $2 b^{\text {loxP/loxP }}$ VE-cadherin $\mathrm{Cre}^{+}$), deletion of Adora $2 b$ on macrophages, monocytes, and granulocytes (Adora2b ${ }^{\text {loxP/loxP }}$ Lysm $\mathrm{Cre}^{+}$), and deletion of Adora $2 b$ on hepatocytes (Adora2 $b^{\text {loxP/loxP }}$ albumin $\mathrm{Cre}^{+}$). As shown in Western blots from isolated endothelial cells, Adora $2 \mathrm{~b}$ expression was significantly attenuated in Adora $2 b^{\text {loxP/loxP }}$ VE-cadherin $\mathrm{Cre}^{+}$mice compared with VE-cadherin $\mathrm{Cre}^{+}$controls (Fig. S5A). Similarly, Western blot analysis for Adora2b expression from isolated macrophages demonstrates that Adora2b expression was significantly attenuated in Adora $2 b^{\text {loxP/loxP }}$ Lysm Cre $^{+}$mice compared with Lysm $\mathrm{Cre}^{+}$controls (Fig. S5B). Finally, Adora2b expression was essentially undetectable in Western blots from isolated hepatocytes derived from Adora $2 b^{\text {loxP/loxP }}$ albumin $\mathrm{Cre}^{+}$compared with controls (Fig. $\mathrm{S} 5 C$ ). Based on previous reports indicating an important role of Adora2b in vascular leakage during hypoxic conditions (19) or during ischemic injury of the kidneys $(12,16)$, we first attempted studies in mice with endothelial Adora $2 b$ deletion. Adora $2 b^{\text {loxP/loxP }} \mathrm{VE}$ cadherin $\mathrm{Cre}^{+}$mice exposed to $45 \mathrm{~min}$ of ischemia followed by $2 \mathrm{~h}$ of reperfusion showed no difference in liver injury as assessed by plasma transaminase levels and liver histology (Fig. 3A). Because Adora2b signaling has been implicated in macrophage functions $(20,21)$, we subsequently exposed $\mathrm{Adora} 2 b^{\text {loxP/loxP }}$ Lysm Cre $\mathrm{Cr}^{+}$mice to $45 \mathrm{~min}$ of ischemia followed by $2 \mathrm{~h}$ of reperfusion. However, we observed no difference in liver injury between the experimental groups and liver histology (Fig. 3B). As the next step, we examined Adora $2 b^{\text {loxP } / l o x P}$ albumin $\mathrm{Cre}^{+}$mice with hepatocellular deletion of Adora2b to similar experimental conditions. Interestingly, these mice demonstrated a similar phenotype as we had previously observed in mice with global deletion of Adora $2 b$. Indeed, we found dramatic increases in liver injury as assessed by plasma transaminase levels and liver histology (Fig. 3C) determined following $45 \mathrm{~min}$ of liver ischemia and $24 \mathrm{~h}$ reperfusion. Together, these studies indicate a hepatocyte-specific role of Adora $2 \mathrm{~b}$ signaling in liver protection from ischemia.

Hepatocyte Adora2b Signaling Attenuates Hypoxic Nuclear Factor NFкB Activation in Vitro. After having shown that Adora2b-dependent liver protection from ischemia and reperfusion predominantly involves hepatocyte-specific Adora2b signaling, we next attempted studies to gain additional insight into the mechanism of Adora2bdependent liver protection. Previous studies had shown that hypoxic conditions such as ischemia and reperfusion are associated with the posttranslational stabilization of NF- $\mathrm{KB}$, leading to a subsequent activation of proinflammatory gene programs (22). A very elegant study from the laboratory of Sean P. Colgan demonstrated that Adora2b signaling in hypoxic lungs represents a means of preventing the activation of NF- $\mathrm{KB}$ during hypoxic conditions (14). Based on these findings, we hypothesized that hepatocyte-specific Adora2b signaling could function as a break for hypoxic NF- $\kappa \mathrm{B}$ activation during hepatic ischemia and reperfusion. To address this hypothesis, we used a lentiviral approach to generate a hepatocyte cell line (HepG2) with siRNA-mediated permanent repression of ADORA2B (Fig. S6 $A$ and $B$ ). When we exposed control cells to conditions of ambient hypoxia $(1 \%$
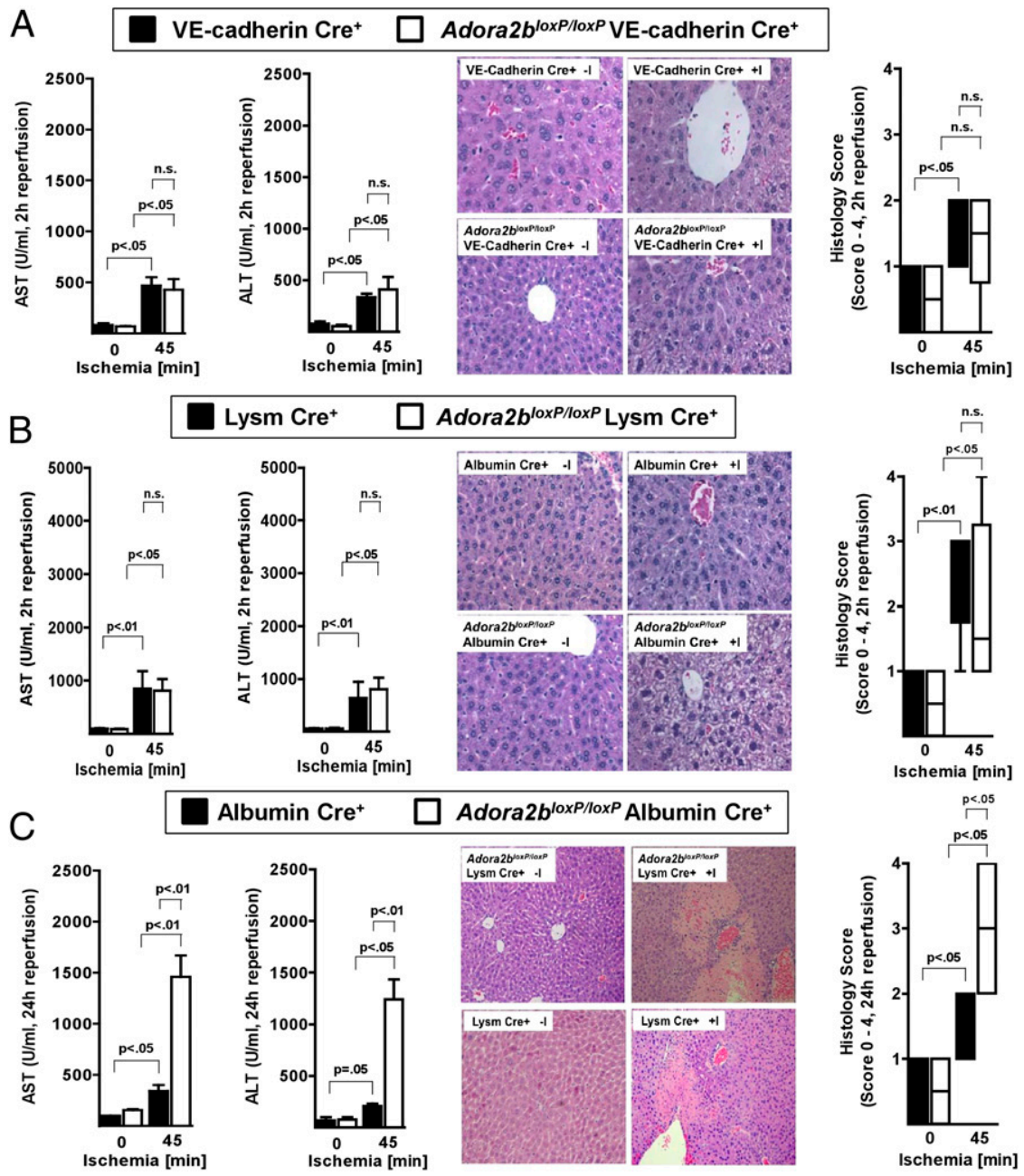

Fig. 3. Consequences of tissue-specific deletion of Adora2b on liver injury due to ischemia. Mice with deletion of Adora2b in $(A)$ vascular endothelia of the liver, Adora2 $b^{\text {IoxPlloxP }}$ VE-vadherin $\mathrm{Cre}^{+}$and VEvadherin $\mathrm{Cre}^{+}$control mice, $(B)$ isolated hepatocytes (Hep) of Adora2b $b^{\text {loxplloxp }}$ Albumin $\mathrm{Cre}^{+}$and Albumin $\mathrm{Cre}^{+}$control mice or (C) macrophages/monocytes/ granulocytes of Adora2 $b^{\text {loxPlloxP }}$ LysmCre $^{+}$and Lysm$\mathrm{Cre}^{+}$control mice were exposed to 45 min of liver ischemia and $2 \mathrm{~h}$ or $24 \mathrm{~h}$ of reperfusion before liver function was assessed by measurement of AST, ALT, liver histology (200-fold magnification; 1 of 3 representative slides is shown), and quantification of histologic tissue injury (Suzuki Scoring Index, 0-4) $(n=3-8)$. 
oxygen, $24 \mathrm{~h}$ ) we observed robust increases in $\mathrm{NF}-\kappa \mathrm{B}$ reporter activity (Fig. $\mathrm{S} 6 C$ ) or its regulatory component $\mathrm{IkB} \alpha$ (Fig. $\mathrm{S} 6 D$ ). This response was significantly increased in cells with siRNAmediated repression of ADORA2B. Moreover, increases of hypoxic NF- $\mathrm{NB}$ or IkB $\alpha$ stabilization were significantly increased following Adora2b inhibition with PSB115, whereas treatment with Adora2b agonist BAY 60-6583 dampened this response. Together, these in vitro studies indicate that hepatocyte-specific ADORA2B signaling attenuates hypoxia-driven activation of proinflammatory programs involving NF- $\mathrm{KB}$.

Pharmacologic Inhibition of NF-кB Restores a "Normal" Phenotype in Gene-Targeted Mice for Adora2b. Encouraged by the above in vitro studies indicating that ADORA2B signaling on hepatocytes can dampen the hypoxic activation of proinflammatory gene programs via inhibition of $\mathrm{NF}-\kappa \mathrm{B}$, we subsequently examined the functional consequences of pharmacologic NF- $\mathrm{kB}$ inhibition during in vivo conditions of hepatic ischemia. For the purpose of these studies, we used a previously described inhibitor of NF- $\kappa B$ (inhibitor of kappa B kinase, 10 mg N-(4-Pyrrolidin-1-yl-piperidin-1-yl)-[4-(4benzo[b]thiophen-2-yl-pyrimidin-2-ylamino)phenyl]carboxamide hydrochloride (IKK-16) i.v. $1 \mathrm{~h}$ before ischemia) (23). Indeed, IKK-16 treatment of mice with global deletion of the Adora2b was associated with the restoration of a phenotype similar to what we had previously observed in wild-type mice, including attenuation of transaminase plasma levels or histologic liver injury (Fig. S7 $A$ ). Moreover, the increased susceptibility of mice with hepatocyte-specific deletion of the Adora2b (Adora $2 b^{\text {loxP/loxP }}$ albumin $\mathrm{Cre}^{+}$) was attenuated (Fig. S7B). Finally, treatment of wild-type mice with IKK-16 was associated with dampened hepatocyte injury as assessed by plasma transaminases and histologic liver injury (Fig. S7C). Taken together, these studies indicate that increased susceptibility of gene-targeted mice for Adora2b to hepatic ischemia and reperfusion injury can be reverted via pharmacologic inhibition of NF- $\kappa$ B. As such, these findings implicate Adora $2 b$ signaling in an endogenous feedback loop to dampen ischemia and reperfusion-elicited increases in hepatocyte inflammation and point toward a therapeutic role for Adora $2 b$ agonists in treating ischemia and reperfusion injury of the liver.

\section{Discussion}

Ischemia and reperfusion injury of the liver represents one of the major challenges during liver surgery and liver transplantation. Due to a lack of donor organs, marginal livers are more frequently used for transplantation. This leads to a dramatic increase in the risk for developing severe ischemia and reperfusion injury during liver transplantation. Therefore, the search for novel pharmacologic strategies to dampen hepatic ischemia and reperfusion injury is currently an area of very intense investigation (2). In the present studies, we pursued the hypothesis that signaling events through any of the four receptors for extracellular adenosine could function as an endogenous feedback look to dampen liver injury during hepatic ischemia and reperfusion. We gained first insight from a small clinical study in patients undergoing liver transplantation. Here, we observed that hepatic transcript and protein levels of ADOAR2B were selectively induced during warm ischemia and subsequent reperfusion. In addition, we were able to recapitulate these findings in a mouse model of hepatic ischemia and reperfusion injury. Moreover, a head-to-head comparison of all four gene-targeted mice for each individual AR during hepatic ischemia and reperfusion injury revealed that $A d o r a 2 b^{-/-}$mice experienced a far more dramatic degree of tissue injury, whereas injury levels in gene-targeted mice for Adora1, Adora2a, or Adora3 were similar to that observed in corresponding control animals. Additional pharmacologic studies with Adora $2 \mathrm{~b}$ antagonist or agonist treatment confirmed a protective role for Adora $2 b$ during hepatic ischemia and reperfusion. Subsequent studies with models of conditional Adora2b deletion indicated that the above observed functional role for Adora2b signaling involves - at least in partsignaling events through Adora $2 b$ receptors expressed on hepatocytes. Moreover, combinations of in vitro and in vivo studies suggest that the observed protection mediated by Adora2b-signaling functions through adenosine-dependent inhibition of hypoxic NK$\kappa \mathrm{B}$ activation. Taken together, the present studies demonstrate what we believe to be a previously unrecognized role for ADORA2B signaling in liver protection from ischemia and reperfusion injury.

In the present studies, we observed a selective induction of Adora2b in patients undergoing liver transplantation or in mice exposed to in situ conditions of hepatic ischemia and reperfusion injury. Indeed, hepatic ischemia and reperfusion injury is characterized by the interdependent relationship of hypoxia and inflammation $(22,24-26)$. In this context, previous studies had examined transcriptional pathways that regulate Adora2b expression during conditions of limited oxygen availability. Indeed, first evidence for hypoxia as a transcriptional regulator of the ADORA2B comes from studies in cultured endothelial cells that were exposed to ambient hypoxia that demonstrated a selective and very robust induction of endothelial ADORA2B transcript and protein levels (27). Other studies identified a previously unknown binding site for the hypoxia-inducible factor (HIF) within the promoter region of human Adora2b (28). Moreover, consistent with the our findings, a recent study in hypoxic preconditioning of the liver found liver protection via Adora2b signaling (29). Indeed, the finding that adenosine signaling can dampen hypoxic inflammation goes back to several landmark papers from the research laboratory of Michael Sitkovsky, that provided the first genetic in vivo evidence for adenosine receptor signaling in dampening inflammation (30) or inflammatory hypoxia (31). Taken together, these studies implicate hypoxia-dependent signaling pathways in the transcriptional control of Adora2b during conditions of limited oxygen availability, and suggest a protective role for such pathways in hepatic ischemia and reperfusion injury.

In addition to the present studies indicating that hepatocytespecific adenosine signaling via Adora $2 b$ is protective, previous studies from the research laboratory of Joel Linden had implicated T-cell-dependent adenosine signaling in protection from ischemia and reperfusion injury $(32,33)$. These studies go back to the finding that inflammatory cells express adenosine receptors that dampen their inflammatory functions $(3,34)$. Other studies with mouse lines deficient in specific populations of lymphocytes demonstrate that $\mathrm{CD}^{+}$and $\mathrm{CD}^{+} \mathrm{T}$ cells play a detrimental role in models of ischemia and reperfusion of the brain (35) or the kidneys (32). Moreover, a very recent study from the laboratory of Mark Okusa revealed that adenosine signaling through dendritic cells expressing Adora2a receptors contributes to attenuating ischemia and reperfusion injury of the kidneys (5). Interestingly, an agonist of ADORA2A (regadenoson) has been approved by the Food and Drug Administration as a coronary vasodilator for patients requiring pharmacologic stress echocardiography (36). Indeed, an ongoing multicenter, dose-finding, and safety trial of infused regadenoson has been initiated to study its safety and efficacy for the treatment of ischemia and reperfusion-related tissue injury in patients with sickle cell disease (37). As such, translational approaches targeting adenosine receptors during liver transplantation might be conceivable in the near future. Whereas there are experimental drugs available as agonists of ADORA2B $(12,38$, 39 ), such compounds have yet to be examined in patients.

The present studies indicate that Adora $2 \mathrm{~b}$ signaling can function as a break for the activation of NK- $\mathrm{KB}$ during hepatic ischemia and reperfusion. Indeed, previous studies have shown that conditions of hypoxia or ischemia and reperfusion are associated with the stabi-

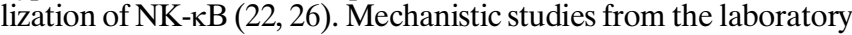
of Cormac Taylor indicate that hypoxia-dependent NK- $\mathrm{KB}$ activation is controlled by oxygen sensing enzymes (prolyl hydroxylase domain proteins, PHDs), and thus shares many similarities with the posttranslational control of HIF protein levels during hypoxia (26). These studies elegantly revealed that PHD1 negatively regulates IKK $\beta$, and hypoxia-associated inhibition of PHD1 subsequently results in the hypoxia-induced activation of NF- $\mathrm{NB}(26)$. Subsequent studies from the laboratory of Sean P. Colgan provided exciting insight into how adenosine signaling through Adora $2 \mathrm{~b}$ can interfere with the hypoxiadependent activation of NK-кB (14). These findings are consistent 
with the present studies showing attenuated NF- $\mathrm{kB}$ activation during hepatic ischemia and reperfusion via Adora $2 \mathrm{~b}$ signaling.

Previous studies have shown that during conditions of hypoxia and ischemia, adenosine is predominantly derived from the breakdown of nucleotides - such as ATP, ADP, and AMP $(9,10$, 39-43). However, there may also be alternative pathways of how extracellular adenosine can be enhanced during conditions of inflammation or ischemia. For example, elegant studies from the laboratory of Edwin Jackson implicate a novel molecular pathway in the extracellular accumulation of adenosine. This pathway includes the extracellular generation of adenosine from $2^{\prime}, 3^{\prime}$-cAMP ("extracellular 2',3'-cAMP-adenosine pathway") (44, 45). Other studies implicate adenosine release through equilibrative nucleoside transporters - for example in the brain or the heart-in elevating extracellular adenosine levels (46-48). Whereas some studies implicate CD39- and CD73-dependent nucleotide metabolism as an important source for hepatic adenosine generation during ischemia $(9,10)$, the contribution of the above discussed pathways in the context of hepatic ischemia and reperfusion injury has to be further examined.

Taken together, the present studies provide unique evidence for a protective role for extracellular adenosine signaling through

1. Eltzschig HK, Sitkovsky M, Robson SC (2012) Purinergic signaling during in flammation. N Engl J Med 367(24):2322-2333.

2. Eltzschig HK, Eckle T (2011) Ischemia and reperfusion-from mechanism to translation. Nat Med 17(11):1391-1401.

3. Cronstein BN, Daguma L, Nichols D, Hutchison AJ, Williams M (1990) The adenosine/ neutrophil paradox resolved: Human neutrophils possess both $\mathrm{A} 1$ and $\mathrm{A} 2$ receptors that promote chemotaxis and inhibit $\mathrm{O} 2$ generation, respectively. J Clin Invest 85(4): $1150-1157$.

4. Rosenberger $P$, et al. (2009) Hypoxia-inducible factor-dependent induction of netrin-1 dampens inflammation caused by hypoxia. Nat Immunol 10(2):195-202.

5. Li L, et al. (2012) Dendritic cells tolerized with adenosine $A_{2} A R$ agonist attenuate acute kidney injury. J Clin Invest 122(11):3931-3942.

6. Faigle M, Seessle J, Zug S, El Kasmi KC, Eltzschig HK (2008) ATP release from vascular endothelia occurs across $\mathrm{C} \times 43$ hemichannels and is attenuated during hypoxia. PLoS ONE 3(7):e2801.

7. Eltzschig HK, Macmanus CF, Colgan SP (2008) Neutrophils as sources of extracellular nucleotides: Functional consequences at the vascular interface. Trends Cardiovasc Med 18(3):103-107.

8. Synnestvedt K, et al. (2002) Ecto-5'-nucleotidase (CD73) regulation by hypoxia-in ducible factor-1 mediates permeability changes in intestinal epithelia. J Clin Invest 110(7):993-1002.

9. Hart ML, Gorzolla IC, Schittenhelm J, Robson SC, Eltzschig HK (2010) SP1-dependent induction of CD39 facilitates hepatic ischemic preconditioning. J Immunol 184(7): 4017-4024.

10. Hart ML, et al. (2008) Extracellular adenosine production by ecto-5'-nucleotidase protects during murine hepatic ischemic preconditioning. Gastroenterology 135(5): 1739-1750.

11. Hart ML, et al. (2008) Use of a hanging-weight system for liver ischemic preconditioning in mice. Am J Physiol Gastrointest Liver Physiol 294(6):G1431-G1440.

12. Grenz A, et al. (2008) The reno-vascular A2B adenosine receptor protects the kidney from ischemia. PLoS Med 5(6):e137.

13. Eckle $T$, et al. (2007) Cardioprotection by ecto-5'-nucleotidase (CD73) and $A 2 B$ adenosine receptors. Circulation 115(12):1581-1590.

14. Khoury J, Ibla JC, Neish AS, Colgan SP (2007) Antiinflammatory adaptation to hypoxia through adenosine-mediated cullin-1 deneddylation. J Clin Invest 117(3):703-711.

15. Liu W, et al. (2011) Sample preparation method for isolation of single-cell types from mouse liver for proteomic studies. Proteomics 11(17):3556-3564.

16. Grenz A, et al. (2012) Equilibrative nucleoside transporter 1 (ENT1) regulates postischemic blood flow during acute kidney injury in mice. J Clin Invest 122(2):693-710.

17. Zhang X, Goncalves R, Mosser DM (2008) The isolation and characterization of murine macrophages. Curr Protoc Immunol Chapter 14:Unit 14.1.

18. Armstrong JM, et al. (2001) Gene dose effect reveals no Gs-coupled A2A adenosine receptor reserve in murine T-lymphocytes: Studies of cells from A2A-receptor-genedeficient mice. Biochem J 354(Pt 1):123-130.

19. Grenz A, Clambey E, Eltzschig HK (2012) Hypoxia signaling during intestinal ischemia and inflammation. Curr Opin Crit Care 18(2):178-185.

20. Hasko G, Csoka B, Nemeth ZH, Vizi ES, Pacher P (2009) A(2B) adenosine receptors in immunity and inflammation. Trends Immunol 30(6):263-270.

21. Németh $\mathrm{ZH}$, et al. (2005) Adenosine augments IL-10 production by macrophages through an $\mathrm{A} 2 \mathrm{~B}$ receptor-mediated posttranscriptional mechanism. J Immuno 175(12):8260-8270.

22. Colgan SP, Taylor CT (2010) Hypoxia: An alarm signal during intestinal inflammation. Nat Rev Gastroenterol Hepatol 7(5):281-287.

23. Waelchli $R$, et al. (2006) Design and preparation of 2-benzamido-pyrimidines as in hibitors of IKK. Bioorg Med Chem Lett 16(1):108-112.

24. Taylor CT (2008) Interdependent roles for hypoxia inducible factor and nuclear factorkappaB in hypoxic inflammation. J Physio/ 586(Pt 17):4055-4059.
ADOAR2B expressed on hepatocytes. Therapeutic extensions of these findings suggest that AORA2B agonists could represent a unique group of pharmacologic agents for the treatment or prevention of hepatic ischemia and reperfusion injury, which would be significant for hepatic transplantations and liver surgery in humans. Whereas these findings are very encouraging, future challenges include the translation of these findings into a clinical setting, particularly with regard to the development of ADORA2B agonists for the treatment of human disease or studies of their safety profile, and their efficiency to prevent or treat ischemia and reperfusion injury during liver transplantation.

ACKNOWLEDGMENTS. We thank Thomas Krahn from Bayer HealthCare AG for kindly providing us with the Adora2b agonist BAY-60-6583, Juergen Schnermann for kindly providing us with his Adora $1^{-1-}$ mice, Marlene Jacobson from Merck Research Laboratories for kindly providing us with her Adora3 $3^{-1-}$ mice, and Joel Linden for kindly providing us with his Adora2a ${ }^{-l-}$ mice. The present research was supported by a Mentored Clinical Scientist Development Award $1 \mathrm{KO}$ KL 103900-01 (to M.A.Z.); an American Heart Association grant (to A.G.); National Heart Institute Grants R01 DK097075, R01-HL0921, R01DK083385, R01- HL098294, and POIHL114457-01; and a grant by the Crohn's and Colitis Foundation of America (to H.K.E.)

25. Taylor CT, Colgan SP (2007) Hypoxia and gastrointestinal disease. J Mol Med (Berl) 85(12):1295-1300.

26. Cummins EP, et al. (2006) Prolyl hydroxylase-1 negatively regulates IkappaB kinasebeta, giving insight into hypoxia-induced NFkappaB activity. Proc Natl Acad Sci USA 103(48):18154-18159.

27. Eltzschig HK, et al. (2003) Coordinated adenine nucleotide phosphohydrolysis and nucleoside signaling in posthypoxic endothelium: Role of ectonucleotidases and adenosine A2B receptors. J Exp Med 198(5):783-796.

28. Kong T, Westerman KA, Faigle M, Eltzschig HK, Colgan SP (2006) HIF-dependent induction of adenosine A2B receptor in hypoxia. FASEB J 20(13):2242-2250.

29. Choukèr $A$, et al. (2012) In vivo hypoxic preconditioning protects from warm liver ischemia-reperfusion injury through the adenosine A2B receptor. Transplantation 94(9):894-902.

30. Ohta A, Sitkovsky M (2001) Role of G-protein-coupled adenosine receptors in downregulation of inflammation and protection from tissue damage. Nature 414(6866):916-920.

31. Thiel M, et al. (2005) Oxygenation inhibits the physiological tissue-protecting mechanism and thereby exacerbates acute inflammatory lung injury. PLOS Bio/ 3(6):e174.

32. Day $Y J$, et al. (2006) Renal ischemia-reperfusion injury and adenosine $2 A$ receptormediated tissue protection: the role of CD4+ T cells and IFN-gamma. J Immunol 176(5):3108-3114.

33. Lappas CM, Day YJ, Marshall MA, Engelhard VH, Linden J (2006) Adenosine A2A receptor activation reduces hepatic ischemia reperfusion injury by inhibiting CD1ddependent NKT cell activation. J Exp Med 203(12):2639-2648.

34. Sitkovsky M, Lukashev D (2005) Regulation of immune cells by local-tissue oxygen tension: HIF1 alpha and adenosine receptors. Nat Rev Immuno/ 5(9):712-721.

35. Yilmaz G, Arumugam TV, Stokes KY, Granger DN (2006) Role of T lymphocytes and interferon-gamma in ischemic stroke. Circulation 113(17):2105-2112.

36. Thompson CA (2008) FDA approves pharmacologic stress agent. Am J Health Syst Pharm 65(10):890.

37. Field JJ, Nathan DG, Linden J (2011) Targeting iNKT cells for the treatment of sickle cell disease. Clin Immunol 140(2):177-183.

38. Schingnitz $U$, et al. (2010) Signaling through the A2B adenosine receptor dampens endotoxin-induced acute lung injury. J Immunol 184(9):5271-5279.

39. Hart ML, Jacobi B, Schittenhelm J, Henn M, Eltzschig HK (2009) Cutting edge: A2B Adenosine receptor signaling provides potent protection during intestinal ischemia/ reperfusion injury. J Immunol 182(7):3965-3968

40. Eckle T, Koeppen M, Eltzschig HK (2009) Role of extracellular adenosine in acute lung injury. Physiology (Bethesda) 24(5):298-306

41. Eltzschig HK (2009) Adenosine: An old drug newly discovered. Anesthesiology 111(4): 904-915.

42. Hart ML, et al. (2011) Hypoxia-inducible factor-1 $\alpha$-dependent protection from intestinal ischemia/reperfusion injury involves ecto- 5 '-nucleotidase (CD73) and the A2B adenosine receptor. J Immunol 186(7):4367-4374.

43. Hart ML, et al. (2008) Role of extracellular nucleotide phosphohydrolysis in intestinal ischemia-reperfusion injury. FASEB $J$ 22(8):2784-2797.

44. Jackson EK, Ren J, Mi Z (2009) Extracellular 2',3'-CAMP is a source of adenosine. J Biol Chem 284(48):33097-33106.

45. Jackson EK, Gillespie DG (2012) Extracellular 2',3'-CAMP-adenosine pathway in proximal tubular, thick ascending limb and collecting duct epithelial cells. Am J Physiol Renal Physiol 304(1):F49-55.

46. Fredholm BB (2012) Rethinking the purinergic neuron-glia connection. Proc Natl Acad Sci USA 109(16):5913-5914.

47. Lovatt $D$, et al. (2012) Neuronal adenosine release, and not astrocytic ATP release, mediates feedback inhibition of excitatory activity. Proc Natl Acad Sci USA 109(16): 6265-6270.

48. Decking UKM, Schlieper G, Kroll K, Schrader J (1997) Hypoxia-induced inhibition of adenosine kinase potentiates cardiac adenosine release. Circ Res 81(2):154-164. 